\title{
Intelligent Graphite Core Condition Monitoring
}

\author{
C.J. Wallace, G.M. West, S.D.J. McArthur and Michael Coghlan* \\ Insitute for Energy and Environment, University of Strathclyde, Glasgow \\ Email: cwallace@eee.strath.ac.uk \\ *GCPT, EDF Energy, Barnwood, Gloucester
}

\begin{abstract}
Condition monitoring of graphite cores has increased significantly in recent years, as the safety cases required to continue operation of Advanced Gas Cooled Reactors (AGR) have evolved. This paper describes the development and implementation of intelligent systems designed to automate and formalise the CM analyses and tasks performed within the remit of the Monitoring Assessment Panels, which are currently being rolled out across the AGR fleet. The implementation of such systems is found to depend crucially on the involvement of station staff at each step of design and implementation, which is highlighted by the successful deployment of second iterations of the IMAPS system for managing reactor observations and the BETA system for automatically analysing refuelling data. Though each system was initially based on an individual analysis or tasks, this paper describes more recent work to allow closer integration of the systems as they are developed, in order to maximise the use of the available data while minimising duplication of effort and required operator time.
\end{abstract}

\section{Keywords}

Monitoring, Analysis, Software

\section{INTRODUCTION}

The introduction of a formal monitoring regime in the Advanced Gas-Cooled Reactor (AGR) fleet was designed to complement increasing inspection as the stations meet, and in some cases exceed, their original design lives but continue to operate through lifetime extension. With monitoring however, came a large increase in the volume of data that required analysis and storage such that station staff could make decisions based on the monitoring information derived from the core. To date, this problem has been tackled in three main strands, of varying maturity and scope, that will form the structure of this paper.

After a brief introduction to the Monitoring Assessment Panels (MAP), the deployment of a system called Intelligent Monitoring Assessment Panels (IMAPS) is described. Now in a second iteration, IMAPS (Jahn, 2007) is used to store and manage Condition Monitoring (CM) observations across the AGR fleet. Emphasis is placed on the interaction with station and central engineering staff to improve the system between the first and second versions of IMAPS. An important component of monitoring regime is the analysis of refuelling data from the AGR, which is performed automatically by a system called BETA (West, 2006), also now in its second revision. Drawing parallels with IMAPS, it is shown that after a successful introduction and after feedback from staff, improvements and refinements were made to a second version of the software.

In the context of the monitoring regime it is shown that IMAPS provides a framework for managing $\mathrm{CM}$ observations, while BETA provides special functionality for a particular analysis. The integration of analyses such as BETA within the IMAPS framework and the addition of further analyses is the topic of the final section of the paper which discusses recent prototyping work using a distributed monitoring system based on intelligent software 
agents and the use of multiple data sets to extract more detailed condition monitoring information. The paper ends with a discussion of future work, with an emphasis on integration.

\section{Monitoring Assessment Panels}

The MAP meetings consider a number of Class 1 parameters (Thermal to Neutron Power Ratios, FGLT analysis, Control Rod monitoring and Fuel Handling observations) as well as a number of Class 2 parameters including various alarms and moderator temperature. Introduced initially at the oldest stations at Hinkley Point B and Hunterston B, the MAP is now being rolled out to other stations across the AGR fleet. As a station owned process, the documentation and analyses vary slightly between stations, however all condition monitoring observations are graded (in order of increasing severity) as blue, green, amber or red.

\section{INTELLIGENT MONITORING ASSESSMENT PANELS}

The initial version of IMAPS was developed and prototyped initially as a standalone application, but after further development and discussion, was deployed as a web application accessible to all users on the EDF network with suitable permissions. Observations are entered by users with the appropriate expertise in a particular parameter and then validated by a different user in order to maintain a high QA grade on the data within the system. The tracking, management and re-grading of observations allows the system to present the user with a view of the data on a particular date, such that decisions made based on the data can be justified in the future.

With this basis for managing and grading observations established, the system was populated with live data and has been used at MAP meetings since 2009. Based on feedback and discussions with EDF Energy staff who used the system, new features and refinements were proposed and discussed along with research focussed work that aims to make maximum use of the available data.

\section{Development and Deployment of Version 2}

Based on discussion with the users of the initial version of IMAPS, several suggestions and new features were discussed, with those which were agreed to most benefit the users prioritised for version 2. As a regularly meeting panel, the MAP documentation varies in content but not in structure between meetings, therefore a templating function was introduced to IMAPS. This facility allows the user to generate data sets, descriptive statistics and summaries of any sub-set of data contained within IMAPS. With a properly structured template document, the MAP forms can be automatically populated before the meeting, provided the data has been uploaded to IMAPS. This should greatly reduce the overhead of paperwork in preparation of MAP meetings and allow for a more straightforward rollout of the system to further stations. IMAPS now also allows the uploading of data from several file types as well as the previous method by which each observation was entered manually by a user. Each observation still requires a second user to validate the information, but using this 'bulk upload' feature, allows for significantly faster addition of data to IMAPS.

As mentioned earlier, the MAP is a station owned process therefor MAP documentation can vary between stations, however there is significant commonality in the work undertaken by 


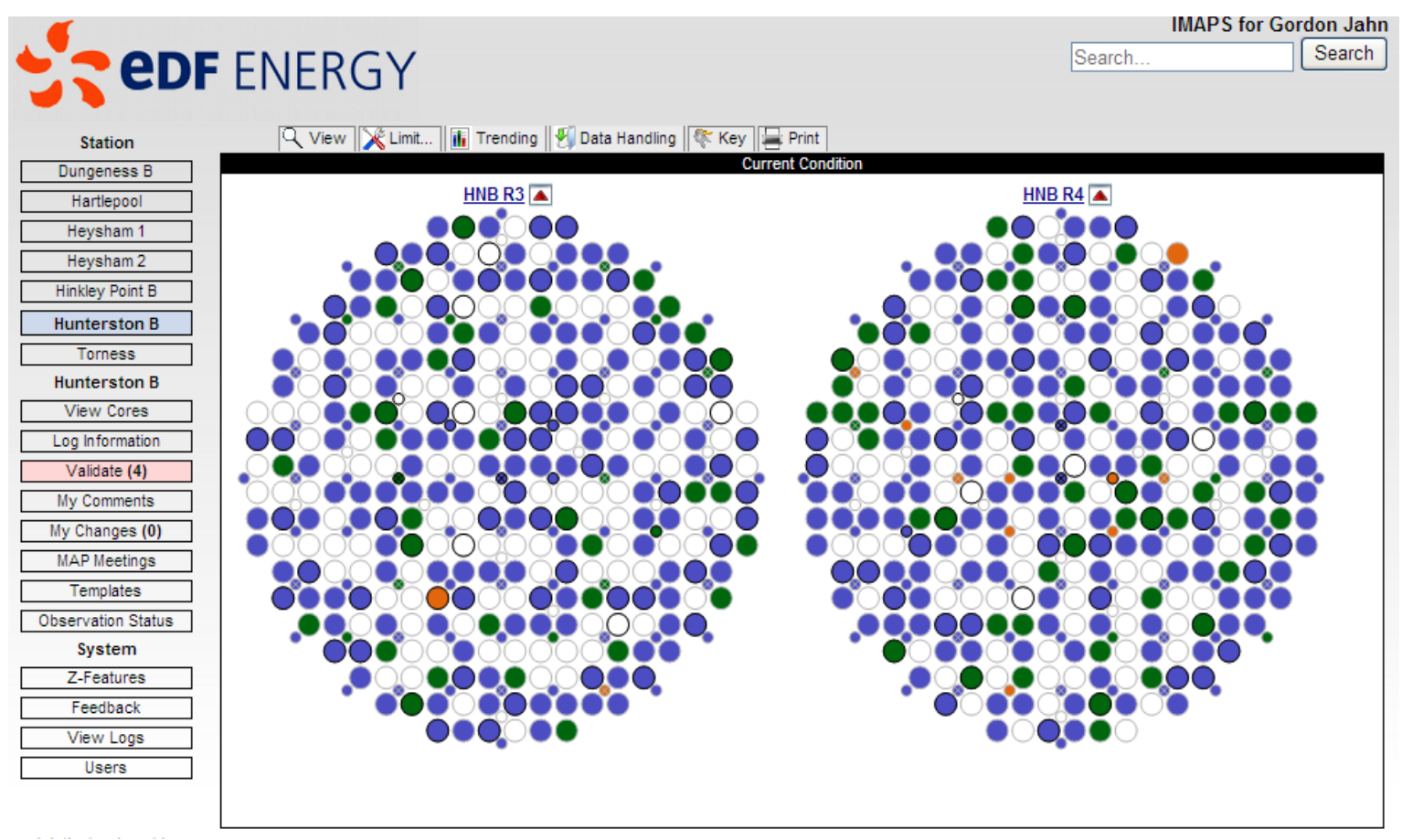

Figure 1 - The IMAPS interface for the Hunterston B reactors, showing the most recent graded data for each component

parameter specialists and the MAP chairman at each meeting. This observation led to the creation of a roles system, whereby an IMAPS user with sufficient permissions can create pre-defined user roles with a sub-set of the available permissions, such that users assigned a role can interact with IMAPS without providing unnescessary access to aspects of IMAPS not required by that user. This feature should allow for an easier adoption of IMAPS as the user base increases, due to the smaller fraction of the system that the user is required to be comfortable with before they use IMAPS on a regular basis. IMAPS version 2 is currently being prepared for deployment by the IMD department at EDF Energy, and will imminently replace IMAPS version 1 on the EDF network.

\section{Trending and Clustering}

As the volume of data stored within IMAPS increases, it was noted that there may be some merit in trending the occurrence of particular event types, grades or observations, therefore the ability to generate plots and statistics based on the data included in the IMAPS database, was included in Version 2. It was noted however, that since the MAP panel grades only observations which were deemed to potentially indicate damage to the core, the total size of the data set within IMAPS is still relatively small, therefore the conclusions which could be drawn from such analyses are currently limited. It is expected however as the MAP process becomes properly defined across the AGR fleet, that useful trends may be detected and improve the understanding of the state and evolution of the core.

As a developmental feature not currently included in IMAPS, clustering was investigated as a method of analysis groups of observations. By comparing the location, time and type of particular observations it is possible to group some observations into clusters, which can be explained by a single root cause. This method of analysis was selected due to the suspicion that it is possible that damage to the core may manifest itself in ways that are perhaps visible earlier in a statistical analysis than on the occasion of direct measurement of a parameter associated with the location of the damage, such as during a refuelling event. 


\section{REFUELLING ANALYSIS}

During refuelling of an AGR, the weight of the fuel assembly is measured as the old fuel is extracted and the new fuel inserted, to ensure that the fuel moves smoothly through the fuel channel and is properly seated in the core. This measurement, and the variation therein, allows the inference of the friction acting upon the fuel as it moves through the internal surface of the fuel channel. The recorded measurement of weight is termed the Fuel Grab Load Trace (FGLT) and following every fuel movement, the FGLT is manually analysed and any potential indicators of core distortion reported formally through the MAP process.

To support the analysis of FGLT, the BETA system was developed which uses an encoded version of the engineer's understanding of refuelling behaviour to automatically interpret the FGLT for the presence of core distortion. The second iteration of the BETA software is now installed at EDF energy and is used to routinely assess refuelling undertaken at both Hunterston B and Hinkley Point B power stations.

\section{Deployment of Version 2}

At the time of writing the BETA database now contains approximately 72,000,000 refueling measurements arising from 1540 fuel movements carried out at either Hunterston B or Hinkley Point B stations. BETA is being used to support the manual analysis of FGLT from both these stations, with expansion to Torness and Heysham 2 expected early in 2012.

Recent work on BETA has focussed on trending of refueling data, where trends in long term graphite shrinkage has been correlated in FGLT measurements (West, 2011).
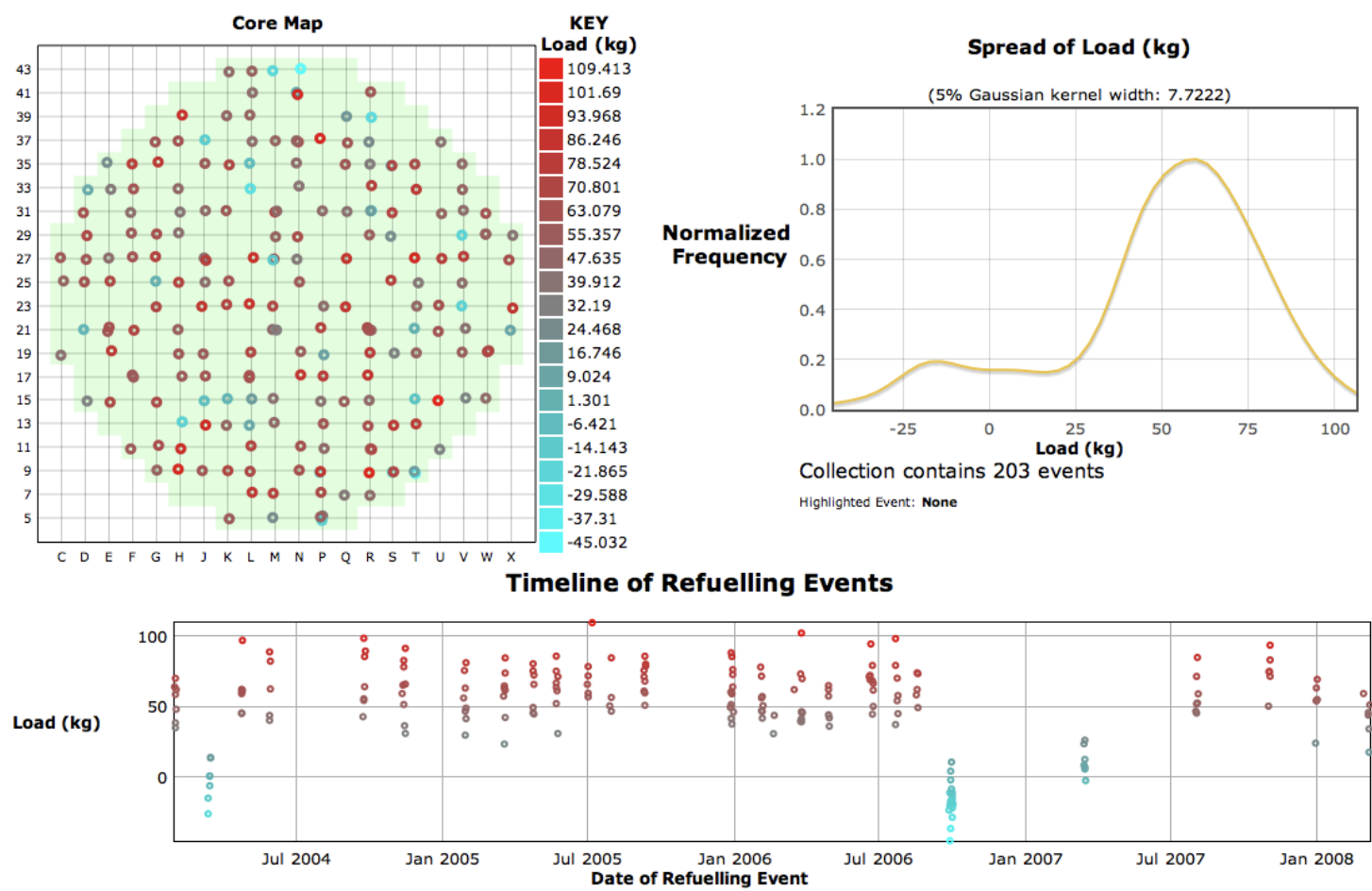

Figure 2 - The BETA interface, showing the trending of loads across the core and with time

Other work has focussed on the deconstruction of the FGLT traces into its constituent sources, allowing the frictional component from the lower stabilizing brushes to be isolated from the rest of the FGLT. This leads to a better representation of the underlying channel 
profile, permitting better detection of anomalies in the masked regions of the trace.

In addition, the data logger at Hunterston B has been upgraded to a new Yokogawa model. In order to manage this transition it is important that the integrity of the data is maintained and that data captured using the new system can still be assessed and compared with the old traces. This was managed within BETA by implementing an additional data reading module at the front end. As a refuelling event is uploaded to BETA, the model of logger is detected and an appropriate conversion of the raw data is undertaken. This ensures that the data held within the BETA database is stored in common units to allow new and historical data to be compared.

Bringing uniformity and interoperability to the various $\mathrm{CM}$ analyses performed within the remit of the MAP has been an important aspect of recent work on BETA and IMAPS. In order to facilitate this work, investigations have begun into the practicability of implementing BETA within a more generic CM framework.

\section{DISTRIBUTED AND INTEGRATE CONDITION MONITORING}

A common feature of monitoring of older industrial equipment is the lack of a modern IT infrastructure, which when combined with the deliberate, nuclear safety guided limitations on networking and IT access of a nuclear power station, significantly complicates the collection, storage and dissemination of reactor data. In order to better resolve this problem, and provide an environment where new analyses or interfaces can be introduced without designing a new system from the ground up, recent work has investigated the viability of implementing a distributed CM system, based on intelligent software agents.

\section{Distributed Agent Framework}

Intelligent agents are small pieces of software which are programmed with particular expertise, such as 'how to analyse refuelling data' or 'how to access and interpret the IMAPS database', and can perform these tasks autonomously. With a community of these agents, which are programmed to be social and can cooperate to perform complex, distributed tasks more efficiently than a human, it is proposed that a distributed monitoring framework could be created. Such a framework would allow for faster and more repeatable condition monitoring analyses, while providing extensibility such that new analyses and data sources can be added as they become available, with only minimal changes to the system.

Systems of many agents, or Multi Agent Systems (MAS) have been used in previous, in power systems and CM applications (Mcarthur, 2007), in a decentralised manner such that the system can be distributed over several physical locations or agent containers as nescessary, but remain federated through network access. Figure 3 shows a proposed structure for such a system, with several containers of agents each with special expertise that they can share with the agent community. Agents can be encoded with domain knowledge that may include how to perform a particular analysis or how to access a particular data type, tasks which it can perform as proxy for any other agent in the system that makes a suitable request.

\section{Ontology}

In order for agents to communicate with each other, they require a common language or ontology. The ontology provides a structure to describe different classes of objects and 


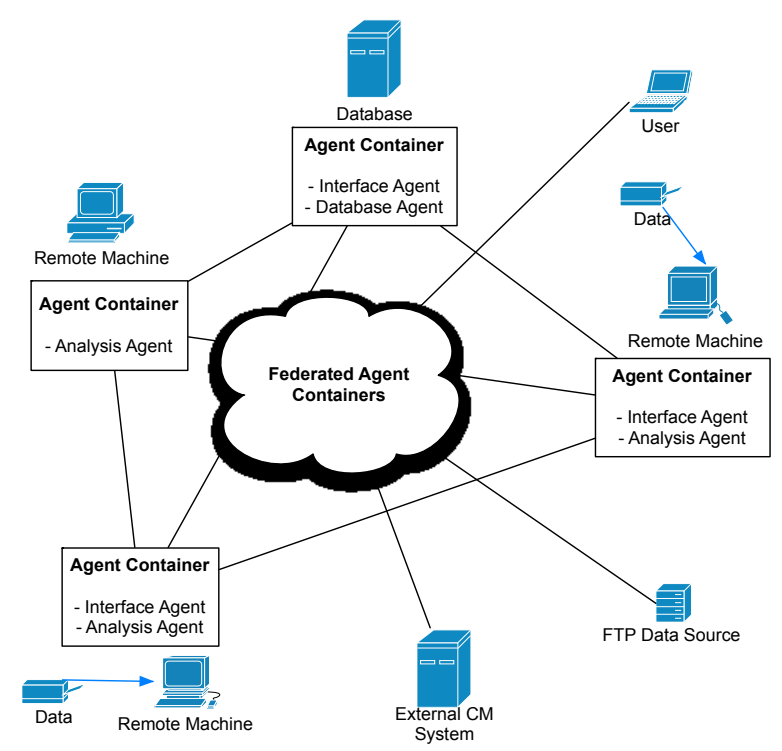

Figure 3 - The proposed structure of a distributed Condition Monitoring system

domain knowledge in the system, such as reactors, control rods and FGLT data as well as allowing reasoning against these objects, for statements such as a fuel channel is within a reactor or low power is less than $x$ percent. Figure 4 shows an example of what such an ontology looks like, showing examples of various physical components in a nuclear power station and how they are related.

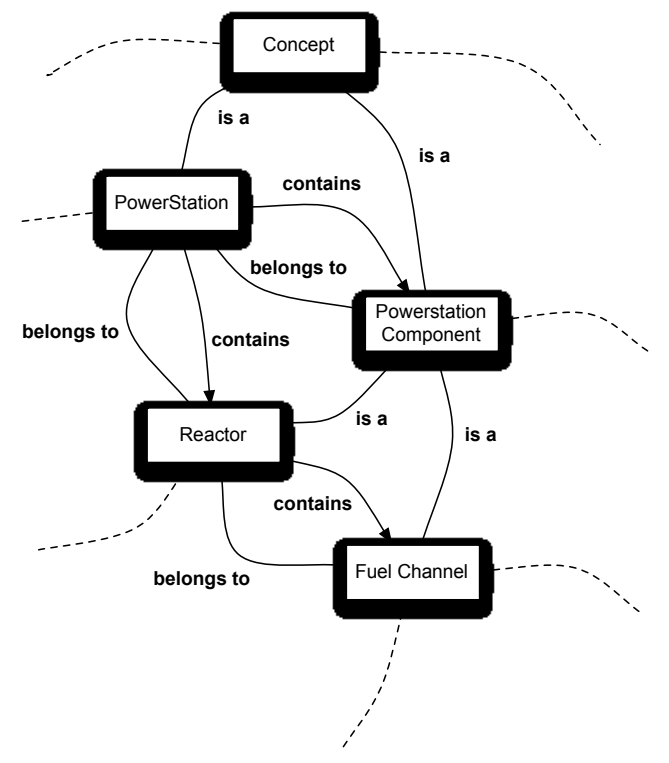

Figure 4 - An example of a part of a nuclear condition monitoring ontology

To demonstrate the suitability of CM analyses to deployment in a MAS, an example of an existing analysis performed as part of the MAP is demonstrated as an analysis agent within the prototype framework.

\section{Thermal and Neutronic Power Analysis}

Regular calculations are made of the channel powers for each AGR core, with a thermal value and a neutronic value calculated for each of approximately 300 channels every 7-10 days. Discrepancies between the two values are often due to modelling errors and core 
operations, such as refuelling or the exchange of control rods however an unexplained discrepancy could potentially indicate damaged fuel that is not being cooled properly. Each time sets of channel powers are generated, an engineer is required to compare the results with all relevant data sets and attempt to correlate events in order to explain the discrepancy. This is a time consuming task, but one that with sufficient data available, is algorithmically suitable to automation for a large percentage of anomalies.
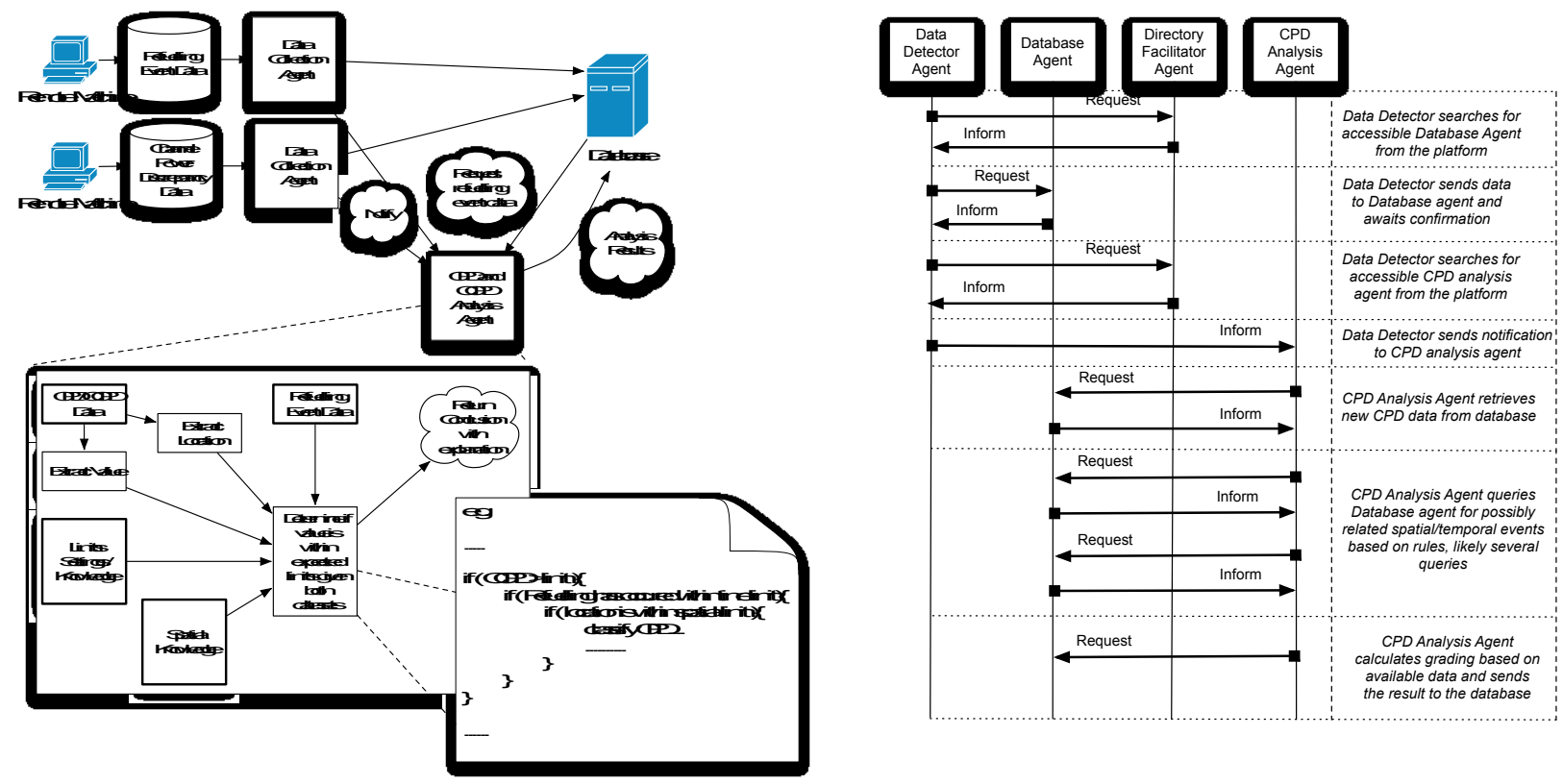

Figure 5 - The process by which a set of agents can perform the channel power analysis, and on the right, the sequence of messages that are passed to perform the analysis

Figure 5 shows how the analysis can be encapsulated in an agent, for the particular case of comparing refuelling records with anomalous channel power discrepancies. The analysis is performed as soon as the data becomes available, takes a fraction of a second and can be performed retrospectively on any amount of relevant data. The results of the analysis are then stored in a database in a form consistent with the ontology and can be used by other analyses or recalled or recalculated later. This example highlights the problem of having several large sets of data from disparate sources with varying availability that require correlation and matching of events, such that the interpretation of an event, in this case an anomalous channel power discrepancy, makes maximum use of available data as quickly as possible.

\section{CONCLUSIONS}

This paper has described the further development of the BETA and IMAPS systems for intelligent CM of the AGR fleet. It was shown that after successful introductions, both systems underwent further development based on feedback and close interaction with station and central engineering staff and a second iteration of each system is now ready to be deployed. The extension of both systems into further analysis of CM data as it is accumulated was shown to be the basis for future work on both systems. As the database of observations in the IMAPS database increases, the application of trending and clustering analysis to the data across the AGR fleet may yield new knowledge of the evolution of the cores, while the application of BETA to analysis of graphite shrinkage has already generated promising results. 
Further analyses and closer integration for both systems has been proposed within a distributed CM framework, for which it suggested than a MAS approach is practical. A demonstration of the encapsulation of an existing MAP analyses within an agent has shown that it is at least possible to create such a framework, however further work is required to determine the practicability of such an installation within an AGR station.

\section{Acknowledgements}

The authors would like to thank EDF Energy for financial support and permission to present this paper. The views expressed in this paper are those of the authors and do not necessarily represent those of EDF Energy.

\section{References}

Jahn, J., McArthur, S.D.J., Reed, J., D.Towle, "Staged Implementation of an Agent Based Advanced Gas- Cooled Reactor Condition Monitoring System”, Proceedings of IEEE Power Engineering Society General Meeting, 2007

West, G.M., Jahn, J., S.D.J.McArthur, McDonald, J. R., Reed, J. “Data Mining Reactor Fuel Grab Load Trace Data to Support Nuclear Core Condition Monitoring", IEEE Transactions on Nuclear Science, Vol 53, No.3 June 2006.

West, G.M., McArthur, S.D.J., Towle, D., "Knowledge-directed characterization of nuclear power plant reactor core parameters", Nuclear Engineering and Design, Volume 241, issue 9, Year 2011, pp. 4013 - 4025.

Mcarthur, S. D. J., Davidson, E. M., Catterson, V. M., Dimeas, A. L., Hatziargyriou, N. D., Ponci, F., \& Funabashi, T. (2007). Multi-Agent Systems for Power Engineering Applications - Part I: Concepts, Approaches, and Technical Challenges. IEEE Transactions on Power Systems, 22(2), 1743-1752.

Mcarthur, S. D. J., Davidson, E. M., Catterson, V. M., Dimeas, A. L., Hatziargyriou, N. D., Ponci, F., \& Funabashi, T. (2007). Multi-Agent Systems for Power Engineering Applications - Part II: Technologies, Standards, and Tools for Building Multi-Agent Systems. IEEE Transactions on Power Systems, 22(4), 1753-1759. 